\title{
Nutritional status, dietary intake and adiposity of normal-weight individuals with clustered metabolic risk factors in the UK population
}

\author{
T. Harrison ${ }^{1}$, F. Amirabdollahian ${ }^{2}$ and I.G. Davies ${ }^{1}$ \\ ${ }^{1}$ School of Sports Studies, Leisure and Nutrition, Liverpool John Moores University, Liverpool, L17 6BD and ${ }^{2}$ School \\ of Health Sciences, Liverpool Hope University, Liverpool, L16 9JD
}

In recent years, a number of novel adiposity proxies, such as the 'A Body Shape Index' (ABSI), Body Roundness Index (BRI) and Waist-hip-height ratio (WHHR) have been applied as an alternative to Body Mass Index (BMI), which has been challenged as proxy indicator of CM risk ${ }^{(1,2)}$. The 'thin outside, fat inside' (TOFI) obesity phenotype is characterised by BMI $18.5-24.9 \mathrm{~kg} / \mathrm{m}^{2}(\mathrm{normal}$ weight), whilst manifesting a cluster of health-detrimental CM abnormalities ${ }^{(3)}$. The aim of this study was to investigate associations between dietary intake, nutritional status and $\mathrm{CM}$ risk in the normal weight UK population with and without clustered $\mathrm{CM}$ risk factors and to verify the suitability of novel adiposity proxies for the identification of TOFIs.

Using the National Diet and Nutrition Survey (NDNS) Rolling Programme 2008/09-2011/12 (unweighted data) a subsample (n 227 ) of the normal weight population was analysed. Variation in nutritional status was investigated by the existence of the cluster $\mathrm{CM}$ risk $^{(4)}$. Beside energy and nutrient intake, emerging (carotenoids) ${ }^{(5)}$ and more established (e.g. plasma Vitamin D, ferritin and dietary carbohydrates ${ }^{(6)}$ correlates of CM risk were investigated. Traditional (waist circumference (WC)) and novel (ABSI, BRI and WHHR) measures of adiposity were also compared amongst groups using an independent t-test.

\begin{tabular}{|c|c|c|c|c|c|c|c|c|}
\hline & \multicolumn{4}{|c|}{ Males (n 78) } & \multicolumn{4}{|c|}{ Females (n 149) } \\
\hline & \multicolumn{2}{|c|}{ TOFI* (n 43) } & \multicolumn{2}{|c|}{ Non-TOFI (n 35) } & \multicolumn{2}{|c|}{ TOFI* (n 49) } & \multicolumn{2}{|c|}{ Non-TOFI (n 100) } \\
\hline & Mean & SD & Mean & SD & Mean & SD & Mean & $\mathrm{SD}$ \\
\hline Age (y) & $43 \cdot 6^{\mathrm{b}}$ & 14.7 & $35 \cdot 23^{\mathrm{b}}$ & 12.8 & $44 \cdot 6$ & $12 \cdot 3$ & 41.8 & $11 \cdot 6$ \\
\hline Weight (kg) & $73 \cdot 0^{\mathrm{a}}$ & $6 \cdot 29$ & $69 \cdot 2^{\mathrm{a}}$ & $7 \cdot 30$ & $60 \cdot 2$ & $5 \cdot 9$ & $60 \cdot 0$ & $6 \cdot 3$ \\
\hline \multicolumn{9}{|l|}{ Nutritional status } \\
\hline Plasma Ferritin (umol/L) & $111 \cdot 03$ & $69 \cdot 5$ & $111 \cdot 18$ & $67 \cdot 8$ & $75 \cdot 0^{\mathrm{b}}$ & $64 \cdot 3$ & $45 \cdot 4^{\mathrm{b}}$ & $36 \cdot 7$ \\
\hline 25-Hydroxy Vitamin D (nmol/L) & $40 \cdot 2^{\mathrm{a}}$ & $25 \cdot 1$ & $53 \cdot 6^{\mathrm{a}}$ & $23 \cdot 6$ & $51 \cdot 4$ & $30 \cdot 4$ & $54 \cdot 1$ & $26 \cdot 8$ \\
\hline Total carotenoids $(\mu \mathrm{mol} / \mathrm{L})$ & $2 \cdot 3$ & $0 \cdot 9$ & $2 \cdot 3$ & $1 \cdot 2$ & $2 \cdot 1^{\mathrm{b}}$ & $0 \cdot 9$ & $2 \cdot 9^{\mathrm{b}}$ & $1 \cdot 3$ \\
\hline \multicolumn{9}{|l|}{ Dietary Intake } \\
\hline Total energy (kcal/d) & $2303 \cdot 9$ & $647 \cdot 9$ & $2169 \cdot 7$ & $490 \cdot 2$ & $1673 \cdot 8$ & $391 \cdot 4$ & $1726 \cdot 2$ & $405 \cdot 3$ \\
\hline Food energy (kcal/d) & $2061 \cdot 1$ & $456 \cdot 2$ & $2099 \cdot 2$ & $466 \cdot 7$ & $1623 \cdot 8$ & $372 \cdot 3$ & $1650 \cdot 5$ & $391 \cdot 1$ \\
\hline Total carbohydrates (\%tot. energy) & $44 \cdot 2^{\mathrm{a}}$ & $8 \cdot 1$ & $47 \cdot 7^{\mathrm{a}}$ & $7 \cdot 5$ & $47 \cdot 2$ & $7 \cdot 5$ & $47 \cdot 4$ & $5 \cdot 8$ \\
\hline Total sugars (\% total energy) & $20 \cdot 2$ & $7 \cdot 3$ & $21 \cdot 1$ & $6 \cdot 7$ & $21 \cdot 4$ & $7 \cdot 9$ & $20 \cdot 2$ & $5 \cdot 2$ \\
\hline NMES (\% total energy) & $13 \cdot 7$ & $6 \cdot 2$ & $13 \cdot 3$ & $6 \cdot 1$ & $11 \cdot 9$ & $7 \cdot 2$ & $10 \cdot 8$ & $4 \cdot 8$ \\
\hline \multicolumn{9}{|l|}{ Adiposity measures } \\
\hline $\operatorname{ABSI}\left(\mathrm{m}^{11 / 6} \mathrm{~kg}^{-2 / 3}\right)$ & $0 \cdot 080$ & $0 \cdot 005$ & 0.078 & $0 \cdot 004$ & 0.077 & $0 \cdot 005$ & 0.076 & $0 \cdot 004$ \\
\hline BRI & $3 \cdot 4^{\mathrm{b}}$ & $0 \cdot 7$ & $2 \cdot 9^{\mathrm{b}}$ & 0.5 & $3 \cdot 1^{\mathrm{a}}$ & 0.8 & $2 \cdot 9^{\mathrm{a}}$ & $0 \cdot 6$ \\
\hline $\mathrm{WC}(\mathrm{cm})$ & $87 \cdot 8^{\mathrm{b}}$ & $6 \cdot 4$ & $82 \cdot 8^{\mathrm{b}}$ & 4.9 & $78 \cdot 8$ & $6 \cdot 3$ & $77 \cdot 0$ & $6 \cdot 0$ \\
\hline WHHR $\left(\mathrm{m}^{-1}\right)$ & $0.51^{\mathrm{a}}$ & $0 \cdot 04$ & $0.48^{\mathrm{a}}$ & 0.03 & $0 \cdot 50^{\mathrm{a}}$ & $0 \cdot 04$ & $0.48^{\mathrm{a}}$ & $0 \cdot 04$ \\
\hline
\end{tabular}

${ }^{\mathrm{a}} \mathrm{p}<0.05^{\mathrm{b}} \mathrm{p}<0.01 ; *$ TOFI $\geqslant 2 \mathrm{CM}$ abnormalities (Triglycerides $>1.7 \mathrm{mmol} / \mathrm{L} ; \mathrm{HDL}<1.0 \mathrm{mmol} / \mathrm{L}(\mathrm{M}) /<1.3 \mathrm{mmol} / \mathrm{L}$ (F) or lipid lowering medication; Systolic/ diastolic blood pressure $\geqslant 130 / 85 \mathrm{~mm} \mathrm{Hg}$ or antihypertensive medication, CRP $>1 \mathrm{mg} / \mathrm{L}$, Glucose $\geqslant 5 \cdot 6 \mathrm{mmol} / \mathrm{L})^{(4)}$.

Female TOFIs had significantly higher levels of plasma ferritin but significantly lower levels of total carotenoids than non-TOFIs. For male TOFIs, plasma Vitamin D was significantly lower than for non-TOFIs. Carbohydrate intake ( \%total energy) was significantly lower in male TOFIs than non-TOFIs. For men only, TOFIs were significantly older and heavier and their BRI, WC and WHHR were significantly higher. For women, only BRI and WHHR were significantly higher in TOFIs. ABSI did not prove to be a useful proxy for adiposity for this group.

Diet quality and composition in this obesity phenotype warrant further investigation to establish how they might impact upon adipose tissue quality, distribution and associated risk factors for CM health. To improve identification of normal-weight individuals at risk, gender specific novel proxy indicators of adiposity may be required.

1 Song X, Jousilahti P \& Stehouwer CD et al. (2013) Eur J Clin Nutr. 67, 1298-1302

2 Thomas DM, Bredlau C \& Bosy-Westphal A et al. (2013) Obesity (Silver Spring) 21, 2264-2271

3 Thomas EL, Frost G \& Taylor-Robinson SD et al. (2012) Nutr Res Rev. 25, 150-61

4 Wildman RP, Muntner P \& Reynolds K et al. (2008) Arch Intern Med. 168, 1617-1624

5 Gammone MA, Riccioni G \& D'Orazio N (2015) Food Nutr Res 59, 26762

6 Mozaffarrian D (2016) Circulation. 133, 187-225 